\section{Neue Wege in der Onkologie und Hämatologie}

Einer Anregung der Redaktion der Schweizerischen Ärztezeitung folgend, möchten wir das vielseitige Programm einer Fortbildungsveranstaltung des Onkozentrums Klinik Im Park (29. März 2000 im Hotel Belvoir, Rüschlikon) mit Referenten aus verschiedenen Zentren in Form von kurzen Zusammenfassungen einem überregionalen Kreis von Interessierten verfügbar machen.

Es war die Absicht der Veranstalter, neue Entwicklungen in der Tumorchirurgie, der internistischen Onkologie und Hämatologie im Rahmen einer Fortbildung einem breiteren Kreis von frei praktizierenden Kolleginnen und Kollegen vorzustellen. Bewusst wurden Themen ausgewählt, die für den Praxisalltag von grosser Bedeutung sein könnten.
Das Programm umfasste chirurgische Themen, welche die Fortschritte in der Behandlung des Rektumkarzinoms und von Lebermetastasen aufzeigen. Weiterhin wurde über den aktuellen Einsatz von monoklonalen Antikörpern in der Behandlung von malignen lymphoproliferativen Erkrankungen berichtet. Ferner wurde eine Neuentwicklung auf dem Gebiet der hämatopoietischen Stammzelltransplantation vorgestellt. Das als "Mini-Transplant" bezeichnete Konzept stellt eine schonende neue Stammzelltherapie dar, welche die T-Zell-vermittelte AntiTumor-Wirkung zu nutzen sucht. Im weiteren wurde über den Einsatz der neuen oralen antihormonellen Medikamente zur Behandlung des Mammakarzinoms diskutiert. Abschliessend wurden die Perspektiven der Onkologie im neuen Jahrtausend vorgestellt.

Prof. Dr. med. Jürg Gmür, PD Dr. med. Victor Hofmann, Zürich 